\title{
The protection of glycyrrhetinic acid (GA) towards acetaminophen (APAP)-induced toxicity partially through fatty acids metabolic pathway
}

\author{
Hua Yang ${ }^{1 \#}$, Tingshu Jiang ${ }^{2 \#}$, Ping $\mathrm{Li}^{1}$, Qishan $\mathrm{MaO}^{2}$
}

1. Respiratory Department, Yantai Hospital of Traditional Chinese Medicine, Xingfu Road No.39,Zhifu District, Yantai City,Shandong Province, China

2. Respiratory Department, Yantai Yuhuangding Hospital Affiliated to Qingdao University; Yuhuangding East Road No.20, Zhifu District, Yantai City,Shandong Province, China

\#These two authors equally contributed to this work.

\begin{abstract}
Background: Acetaminophen (APAP)-induced liver toxicity remains the key factor limiting the clinical application of APAP, and herbs are the important sources for isolation of compounds preventing APAP-induced toxicity.

Aims: To investigate the protection mechanism of glycyrrhetinic acid towards APAP-induced liver damage using metabolomics method.

Methods: APAP-induced liver toxicity model was made through intraperitoneal injection (i.p.) of APAP (400 mg/kg). Glycyrrhetinic acid was dissolved in corn oil, and intraperitoneal injection (i.p.) of glycyrrhetinic acid ( $500 \mathrm{mg} / \mathrm{kg}$ body weight) was performed for 20 days before the injection of APAP. UPLC-ESI-QTOF MS was employed to analyze the metabolomic profile of serum samples.
\end{abstract}

Results: The pre-treatment of glycyrrhetinic acid significantly protected APAP-induced toxicity, indicated by the histology of liver, the activity of ALT and AST. Metabolomics showed that the level of palmtioylcarnitine and oleoylcarnitine significantly increased in serum of APAP-treated mice, and the pre-treatment with GA can prevent this elevation of these two fatty acid-carnitines.

Conclusion: Reversing the metabolism pathway of fatty acid is an important mechanism for the protection of glycyrrhetinic acid towards acetaminophen-induced liver toxicity.

Keywords: Glycyrrhetinic acid (GA), acetaminophen (APAP), metabolomics, fatty acid

DOI: http://dx.doi.org/10.4314/ahs.v15i3.42

Cite as: Yang H, Jiang T, Li P, Mao Q. The protection of glycyrrhetinic acid (GA) towards acetaminophen (APAP)-induced toxicity partially through fatty acids metabolic pathway. Afri Health Sci. 2015;15(3):1023-7. doi: http:// dx.doi.org/10.4314/abs.v15i3.42

\section{Introduction}

The human respiratory system is divided into the upper

\author{
Corresponding author: \\ Hua Yang, \\ Respiratory Department, \\ Yantai Hospital of Traditional \\ Chinese Medicine, \\ Xingfu Road No.39, \\ Zhifu District,Yantai City, \\ Shandong Province, China \\ Qishan Mao, \\ Respiratory Department, \\ Yantai Yuhuangding Hospital Affiliated \\ to Qingdao University; \\ Yuhuangding East Road No.20, \\ Zhifu District, Yantai City, \\ Shandong Province, China; \\ E-mail: jiangshandong123@163.com
}

and lower respiratory tract. The upper respiratory tract comprises the nasal cavity, pharynx, and larynx, and the lower respiratory tract is consisted of the trachea, bronchi, and the lungs. The respiratory system diseases contain upper respiratory tract infections, lower respiratory tract infections, asthma, pneumothorax, chronic obstructive pulmonary disease, pulmonary fibrosis, tuberculosis, and even cancers ${ }^{1,2}$. Searching effective drugs for respiratory system diseases is very important. Glycyrrhetinic acid, also called as enoxolone, is a pentacyclic triterpenoid derivative of the beta-amyrin type obtained from the hydrolysis of glycyrrhizic acid which is an important herbal ingredient isolated from herb licorice $^{3}$. Glycyrrhetinic acid has exerted therapeutic role towards respiratory diseases. For example, glycyrrhetinic acid has been demonstrated to show therapeutic role towards non-small cell lung cancer ${ }^{4}$.

The adverse effects of acetaminophen (APAP) remain the major reason in limiting the clinical application of 
APAP which is one of the most important over-thecounter analgesic and antipyretic drugs. The reason for APAP-induced adverse effects is the bioactivation of APAP into the reactive metabolite $\mathrm{N}$-acetyl-p-benzoquinone imine (NAPQI) which is the key substance basis for APAP-induced toxicity . Metabolomics-based system biology method has been widely used to elucidate the detailed mechanism of APAP-induced liver toxicity. The experiment performed by Chen et al. used metabolomics method to compare the metabolism behavior of APAP between wild type and CYP2E1-null mice, and found several new metabolites of APAP among which APAP dimer has high correlation with the toxicity of APAP5. Serum metabolomics analysis showed that APAP induced mitochondria damage through disrupting oxidative process of fatty acids ${ }^{6}$. Furthermore, the protective role of PPAR alpha agonist towards APAP-induced toxicity supported the importance of fatty acid metabolism in APAP-induced toxicity ${ }^{7}$.

Given that the disruption of fatty acids metabolism is an important reason for APAP-induced toxicity, the present study aimed to investigate the influence of glycyrrhetinic acid towards acetaminophen (APAP)-induced fatty acids metabolism.

\section{Materials and methods \\ Chemicals and reagents}

Acetaminophen (APAP), acetonitrile, and formic acid were purchased from Sigma-Aldrich (St. Louis, Mo., USA).

\section{Animal experiment and sample preparation}

The animal experiment was performed under the agreement approved by the committee of Yantai Yuhuangding Hospital. 12 Male Sv/129 mice were purchased from Beijing Laboratory Animal Research Center (Beijing, China). The mice were maintained under a standard 12-h light/12-h dark cycle with food and water ad libitum. APAP-induced liver toxicity model was made through intraperitoneal injection (i.p.) of APAP (400 $\mathrm{mg} / \mathrm{kg}$ ). Glycyrrhetinic acid was dissolved in corn oil, and intraperitoneal injection (i.p.) of glycyrrhetinic acid (500 mg/kg body weight) was performed for 20 days before the injection of APAP. Blood was collected at 6 hour after the administration of APAP using BD MicrotainerTM serum separator tubes (Franklin Lakes, NJ) by retro-orbital bleeding. After the sacrifice of mice, the livers were excised and used for histology analysis.

\section{Determination of activity of aspartate aminotrans- ferase (AST) and alanine transaminase (ALT)}

The mechanism for determination of AST and ALT activity is to the determination of reaction velocity for the catalysis process of oxidation of NADH to NAD+. During this process, $200 \mu \mathrm{L}$ of ALT or AST assay buffer was added to catalyze the reaction of $1 \mu \mathrm{L}$ of serum. The whole catalytic process was monitored using the wavelength at $340 \mathrm{~nm}$.

\section{Serum metabolomics analysis}

$10 \mu \mathrm{L}$ of serum was firstly mixed with $190 \mu \mathrm{L}$ of $80 \%$ acetonitrile, and the centrifugation at $14,500 \mathrm{rpm}$ was used to deproteinization. $5 \mu \mathrm{L}$ of the supernatant was added into the vials to undergo UPLC-QTOF-MS analysis. Acquity UPLC BEH C18 column $(1.7 \mu \mathrm{m}, 2.1 \times 50$ $\mathrm{mm}$, Waters Corp.) was used to seperate the compounds. The elution phase was consisted of water with $0.2 \%$ formic acid (A) and acetonitrile with $0.5 \%$ formic acid (B). The gradient elution condition was used as followed: $0-3 \mathrm{~min}, 98 \%-70 \% \mathrm{~A}$; 3-8 $\mathrm{min}, 70 \%-5 \%$ A; $8-9 \mathrm{~min}, 5 \%$ A. Finally, the elution condition was brought back to $98 \%$ A. The column temperature was maintained at $40^{\circ} \mathrm{C}$. Positive mode was used to monitor all the compounds, and the capillary and cone voltages were set as $4 \mathrm{kV}$ and $30 \mathrm{~V} .120$ and $360^{\circ} \mathrm{C}$ were selected as source and desolvation temperature, respectively. The elution rate of nitrogen gas was set as $50 \mathrm{~L} / \mathrm{h}$ and $600 \mathrm{~L} / \mathrm{h}$ for cone gas and desolvation gas, respectively. For MS/MS fragmentation, the collision energy ranged from 10 to $40 \mathrm{eV}$.

\section{Statistical method:}

The statistical analysis was performed using twotailed one student's $t$ test.

\section{Results}

The Figure 1 gave the phenotype results. As shown in Figure 1A, compared with the histology of control mice, the livers from APAP-treated mice had some damaged areas, as indicated using arrow sign. The pretreatment with GA can protect the damage induced by APAP treatment (Figure 1A). 
Figure 1 Pre-treatment with glycyrrhetinic acid (GA) significantly protected acetaminophen (APAP)-induced liver toxicity. (A) H\&E staining to analyze the liver histology from control group, APAP-treated group and GA+APAP-treated group. (B) Comparison of ALT activity in control group, APAP-treated group and GA+APAP-treated group. ***, $\mathbf{P}<0.001$. (C) Comparison of AST activity in control group, APAP-treated group and GA+APAP-treated group. $* * *, \mathbf{P}<0.001$.

\section{Figure 1}

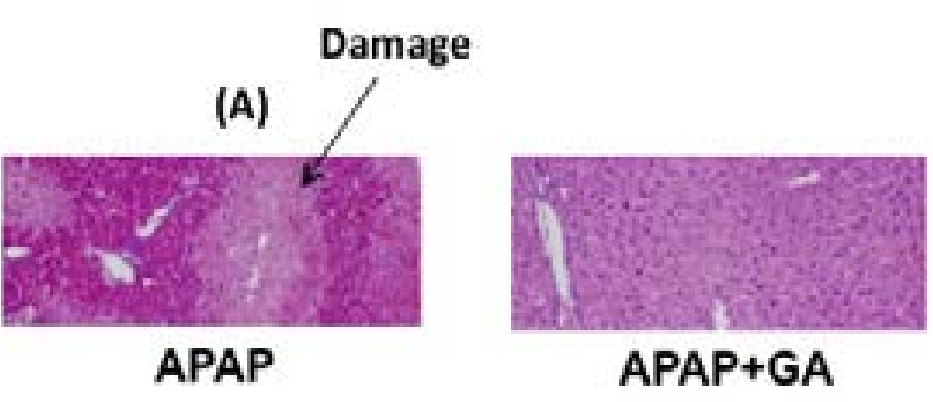

(B)

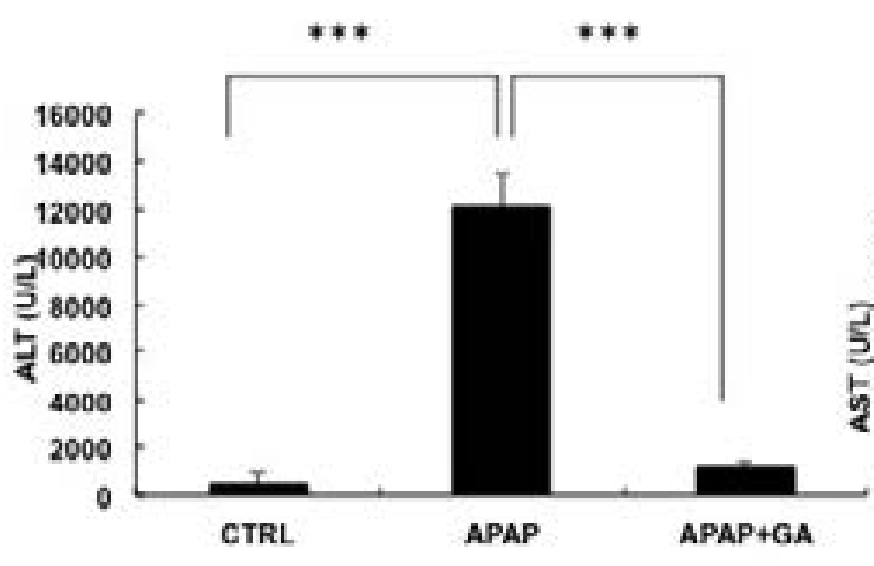

\section{(C)}

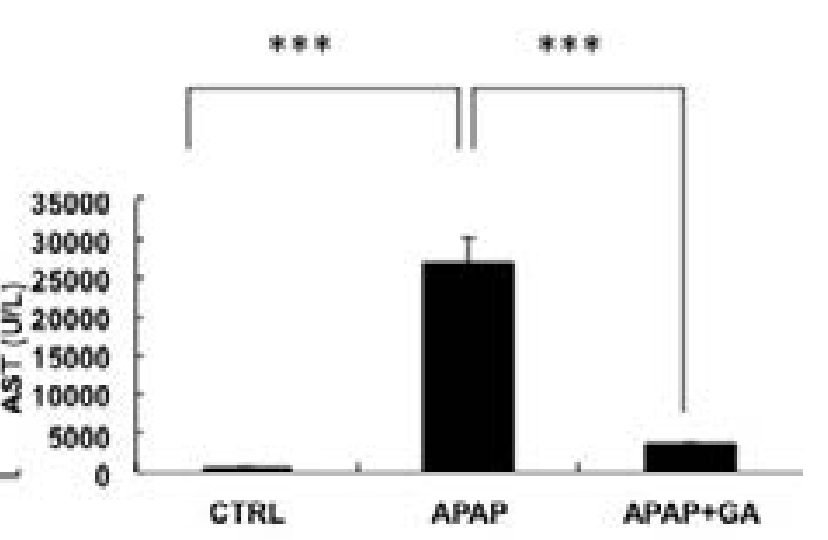

The pre-treatment with GA also prevented the elevation of ALT and AST activity induced by APAP treatment (Figure 1B \&C).

The treatment with APAP significantly increased the level of two ions in serum, and these two ions were identified to be palmtioylcarnitine and oleoylcarnitine (Supplemental Figure 1). The treatment with APAP significantly induced the elevated level of these two compounds $(p<0.001$, Figure 2$)$, and pre-treatment with GA can significantly decrease the level of these two compounds in serum $(p<0.001$, Figure 2$)$. 


\section{Figure 2}

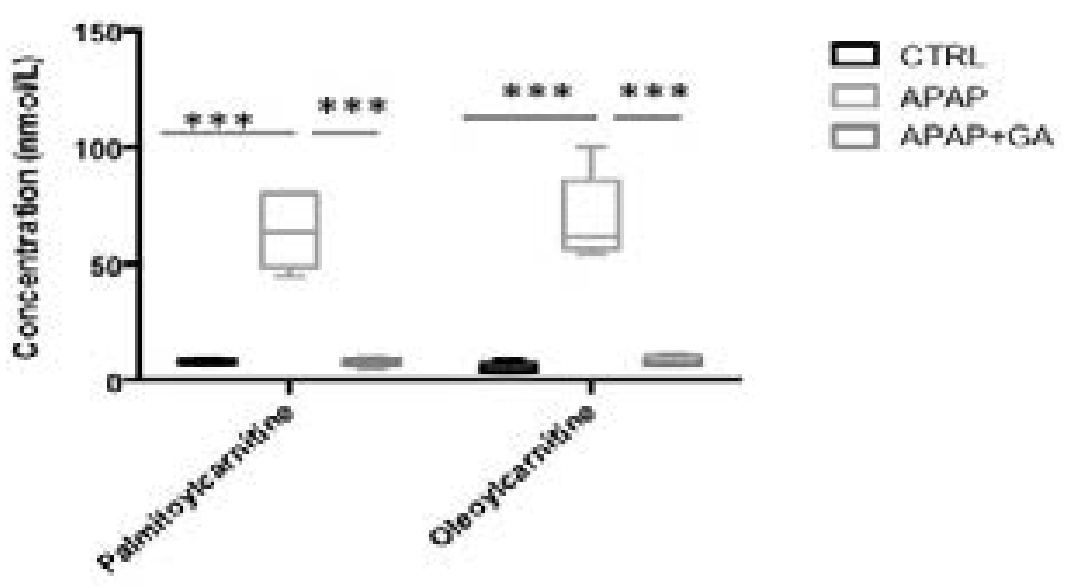

\section{Discussion}

Herbs have become more and more popular in the world, and herbs have exerted therapeutic role towards more and more diseases. The therapeutic behavior of herbs towards APAP-induced liver toxicity has been frequently reported. For example, herb Schisandra Sphenanthera extract has been demonstrated to exhibit therapeutic role towards APAP-induced liver toxicity ${ }^{8}$, and the compound isolated from this herb Schisandrol $\mathrm{B}$ has also exerted its protection role towards APAP-induced liver toxicity ${ }^{9}$. Herbal ingredient saikosaponin d (SSd), isolated from traditional Chinese herb Bupleurum falcatum, protects against APAP-induced hepatotoxicity by inhibiting NF-kB and STAT3 signaling ${ }^{10}$.

The present study demonstrated the therapeutic role of glycyrrhetinic acid towards APAP-induced liver toxicity through reversing fatty acids metabolic pathway. Given that fatty acids metabolism is a key source for energy production, glycyrrhetinic acid-induced recovery of energy production might be the key mechanism for the protection of glycyrrhetinic acid towards APAP's toxicity. Targeted metabolomics analysis demonstrated that glycyrrhizin protected APAP-induced liver toxicity through reversing fatty acid metabolism ${ }^{11}$. Therefore, the same protection mechanism of the compounds with such similar structures might exist. It should be noted that glycyrrhetinic acid (GA) can inhibit the activity of CYP2E1 which might also contribute to the protection of GA towards APAP-induced toxcitiy ${ }^{12}$.

\section{Conclusion}

Reversing the metabolism pathway of fatty acid is an important mechanism for the protection of glycyrrhetinic acid towards acetaminophen-induced liver toxicity.

\section{References}

1.Jenkins $G$, Goodwin A. Novel approaches to pulmonary fibrosis. Clin Med 2014; Suppl 6: s45-s49.

2. Froesch P, Martucci F, Gyorik S, Dutly AE, Cafarotti $\mathrm{S}$. Management of non-small cell lung cancer in the elderly. Eur J Intern Med 2014; 25(10): 888 PubMed -894. 3. AsI MN, Hosseinzadeh H. Review of pharmacological effects of Glycyrrhiza sp. and itsbioactive compounds. Phyther Res 2008; 22(6): 709 PubMed -724.

4. 18b-Glycyrrhetinic acid suppresses cell proliferation through inhibiting thromboxane synthase in non-small cell lung cancer. PLoS One 2014; 9(4): PubMed e93690. 5. Chen C, Krausz KW, Idle JR, Gonzalez FJ. Identification of novel toxicity-associated metabolites by me- 
tabolomics and mass isotopomer analysis of acetaminophen metabolism in wild-type and Cyp2e1-null mice. J Biol Chem 2008; 283(8):4543 PubMed -4559.

6. Chen C, Krausz KW, Shah YM, Idle JR, Gonzalez FJ. Serum metabolomics reveals irreversible inhibition of fatty acid beta-oxidation through the suppression of PPARalpha activation as a contributing mechanism of acetaminophen-induced hepatotoxicity. Chem Res Toxicol 2009; 22(4):699 PubMed -707.

7. Patterson AD, Shah YM, Matsubara T, Krausz KW, Gonzalez FJ. Peroxisome proliferator-activated receptor alpha induction of uncoupling protein 2 protects against acetaminophen-induced liver toxicity. Hepatology 2012; 56(1): 281 PubMed -290.

8. Fan X, Jiang Y, Wang Y, Tan H, Zeng H, Wang Y, Chen P, Qu A, Gonzalez FJ, Huang M, Bi H. Wuzhi tablet (Schisandra Sphenanthera extract) protects against acetaminophen-induced hepatotoxicity by inhibition of CYP-mediated bioactivation and regulation of NRF2ARE and p53/p21 pathways. Drug Metab Dispos 2014; 42(12): 1982 PubMed -1990.
9. Jiang $\mathrm{Y}$, Fan X, Wang $\mathrm{Y}$, Chen $\mathrm{P}$, Zeng $\mathrm{H}$, Tan $\mathrm{H}$, Gonzalez FJ, Huang M, Bi H. Schisandrol B protects against acetaminophen-induced hepatotoxicity by inhibition of CYP-mediated bioactivation and regulation of liver regeneration. Toxicol Sci 2015; 143(1): $107 \mathrm{Pu}-$ bMed -115 .

10. Liu A, Tanaka N, Sun L, Guo B, Kim JH, Krausz KW, Fang Z, Jiang C, Yang J, Gonzalez FJ. Saikosaponin $\mathrm{d}$ protects against acetaminophen-induced hepatotoxicity by inhibiting NF-kB and STAT3 signaling. Chem Biol Interact 223C: 80-86.

11. Yu J, Jiang YS, Jiang Y, Peng YF, Sun Z, Dai XN, Cao QT, Sun YM, Han JC, Gao YJ. Targeted metabolomics study indicating glycyrrhizin's protection against acetaminophen-induced liver damage through reversing fatty acid. Phytother Res 2014; 28(6): 933 PubMed -936. 12. Jeong HG, You HJ, Park SJ, Moon AR, Chung YC, Kang SK, Chun HK. Hepatoprotective effects of 18beta-glycyrrhetinic acid on carbon tetrachloride-induced liver injury: inhibition of cytochrome P450 2E1 expression. Pharmacol Res 2002; 46(3): 221 PubMed -7. 